blocking complement-dependent injury thereby reducing risk of lung transplant rejection. OBJECTIVES/GOALS: Our goal was to determine if pre-existing autoimmune autoantibodies, such as those resulting from cigarette smoke (CS), contribute to graft rejection in lung transplantation (LTx) and if autoreactive-mediated graft injury is complement-dependent. METHODS/STUDY POPULATION: For in vivo experiments, we utilized our emphysema mouse model. Briefly, eight-week-old C57BL/6J mice are exposed to 3R4F reference cigarette smoke 5 hours per day, 5 days a week for 6 months. Upon completion, cigarette smoked (CS) mice and control (NS) mice received syngeneic orthotopic left-lung transplant from age-matched C57BL/6J donors. To determine if pre-existing autoreactivity mediated graft injury was complement-dependent we treated CS-LTx mice with a novel, bifunctional complement inhibitor. Autoantibody levels were measured by ELISA and lung injury was assessed by blinded histopathological analyses. Complement inhibition was verified by immunofluorescence. RESULTS/ ANTICIPATED RESULTS: We found that CS-exposure leads to production of autoreactive antibodies towards extracellular matrix (ECM) components and contributes to graft injury. Interestingly, LTx into CS exposed mice further increased de-novo ECM autoantibody development. Lastly, treatment with our novel, bifunctional complement inhibitor blocked autoantibody spreading and significantly reduced graft rejection. DISCUSSION/SIGNIFICANCE OF FINDINGS: These data demonstrate that smoking induces preLTx autoreactivity to ECM proteins that promotes graft injury following LTx. Furthermore, complement inhibition reduces autoantibody production and protects the graft from injury.

89723

\section{CD105-targeted CAR T cells for the treatment of acute} myeloid leukemia*

Konstantinos Lontos, Yiyang Wang, Andrew Frisch, Mason Colbert, Jason Lohmueller and Greg M. Delgoffe

${ }^{1}$ University of Pittsburgh and ${ }^{2}$ Tsinghua University

ABSTRACT IMPACT: Our work might lead to a new treatment for patients with acute myeloid leukemia OBJECTIVES/GOALS: Acute myeloid leukemia (AML) is a devastating hematologic malignancy, with dismal 5-year survival. Chimeric antigen receptor (CAR) T cells have been approved for B cell malignancies but not for AML. The goal of this study is to explore the safety and efficacy of CAR T cells targeting CD105 (endoglin) to treat AML. METHODS/STUDY POPULATION: We have constructed human and murine CAR T cells targeting CD105. The CARs were created by sequencing the $\mathrm{V}(\mathrm{D}) \mathrm{J}$ regions of hybridomas and designing single chain variable fragments that target CD105 which were subsequently introduced in a CAR backbone via Gibson assembly. The CAR T cells were produced via transduction using retrovirus or lentivirus. Leukemia cell lines were assessed for CD105 expression with flow cytometry. Killing assays were performed via measurement of luminescence of target cells after co-culture with CAR T cells. Activation assays were performed with co-culture of CAR $\mathrm{T}$ cells and target cells and measurement of activation markers with flow cytometry. To assess in vivo efficacy and safety, murine CAR T cells were infused into C57BL/6J mice carrying B16 melanoma after lymphodepletion. RESULTS/ANTICIPATED RESULTS: All human leukemia cell lines assessed (Nalm6, MOLM-14, MV4-11, Kasumi-1, THP-1) expressed some degree of endoglin apart from the $\mathrm{T}$ cell leukemia Jurkat. Human CD105 CAR T cells were activated by co-culture with leukemia cell lines and effectively killed leukemia cells in vitro in a
CD105-specific manner. Murine CAR T cells killed efficiently both murine solid tumors (B16 melanoma) and murine leukemias (C1498) in vitro. Murine CAR T cells did not exhibit any toxicity when infused after low-dose lymphodepletion (cyclophosphamide $100 \mathrm{mg} / \mathrm{kg}$ ) but caused significant morbidity after higher doses (cyclophosphamide $200 \mathrm{mg} / \mathrm{kg}$ ). Murine CAR T cells delayed the growth of B16 melanoma in immunocompetent mice. DISCUSSION/SIGNIFICANCE OF FINDINGS: We have constructed human and murine CD105 CAR T cells with excellent activity in vitro. The activity of human CD105 CAR T cells in xenografts and the biologic relevance of the toxicity of murine CD105 CAR T cells in humans needs to be further investigated. CD105 CAR T cells might prove an important therapeutic option for patients with AML.

91074

\section{Identification of monoclonal antibodies with broad reactivity against the malaria parasite variant surface antigen responsible for severe malaria ${ }^{\dagger}$}

Raphael Reyes ${ }^{1}$, Louise Turner ${ }^{2}$, Isaac Ssewanyana ${ }^{3}$, John Rek ${ }^{3}$, Bryan Greenhouse ${ }^{4}$, Sebastiaan Bol ${ }^{1}$, Thomas Lavstsen ${ }^{2}$ and Evelien M. Bunnik ${ }^{1}$

${ }^{1}$ Department of Microbiology, Immunology and Molecular Genetics, The University of Texas Health Science Center, San Antonio, TX, USA, ${ }^{2}$ Faculty of Health and Medical Sciences, University of Copenhagen, Copenhagen, Denmark, ${ }^{3}$ Infectious Disease Research Collaboration, Kampala, Uganda and ${ }^{4}$ Department of Medicine, University of California San Francisco, San Francisco, CA, USA

ABSTRACT IMPACT: This study aims to provide insight into naturally acquired immunity against severe malaria, thereby laying the foundation for the design of novel vaccine candidates to prevent severe disease as well as monoclonal antibody therapies to treat severe malaria. OBJECTIVES/GOALS: Severe malaria is caused by parasite surface antigens that contain high sequence diversity. Nevertheless, P. falciparum-exposed individuals develop antibody responses against these antigens. Our goal is to isolate antibodies with broad reactivity to understand how disease protection is acquired. METHODS/STUDY POPULATION: Our study cohort consists of Ugandan adults living in a malaria-endemic region with high transmission intensity, who are protected against severe malaria. Using fluorescently labeled probes of parasite surface antigens, we have isolated antigen-specific B cells from these donors. We then expressed the corresponding monoclonal antibodies in vitro. These antibodies were screened against a library of variant surface antigens to determine antibody breadth and potential to inhibit interaction of the parasite surface antigen with host receptors, a critical step in pathogenesis. Additionally, using a panel of variant surface antigen mutants, we have predicted the epitopes targeted by the broadest monoclonal antibodies. RESULTS/ANTICIPATED RESULTS: We have identified three monoclonal antibodies with exceptionally broad reactivity and inhibitory activity against our panel of severe disease-inducing variant surface antigens. We have identified two major sites targeted by these broadly reactive antibodies. The first site was associated with the largest breadth, but limited inhibitory potential, while the second site showed high-affinity antibody binding and inhibition of receptor binding. Interestingly, two of these three antibodies were very similar in structure, even though they were isolated from different donors. Isolation of antigen-specific B cells from additional donors will enable us to identify how common such broadly reactive antibodies are and allow the identification of additional epitopes DISCUSSION/SIGNIFICANCE OF 